\title{
Schrödinger and Planck oscillators: not quite the same physics for a modified Einstein solid
}

\author{
Enrique N. Miranda \\ Facultad de Ciencias Exactas y Naturales, Universidad Nacional de Cuyo, Mendoza, Argentina. \\ IANIGLA, CONICET, CCT Mendoza, Argentina. \\ Departamento de Física, Universidad Nacional de San Luis, San Luis, Argentina. \\ e-mail:emiranda@mendoza-conicet.gov.ar
}

Received 20 January 2020; accepted 5 February 2020

\begin{abstract}
In the statistical mechanics of quantum harmonic oscillators, the zero-point energy can either be included (Schrödinger oscillators) or omitted (Planck oscillators). For the usual results, the type of oscillator makes no difference but, looking more closely, it turns out that including or not this energy is not without consequences. A simple model called modified Einstein solid (MES) is introduced. In this model, the frequency of the oscillators changes with the volume of the solid, and this change is characterized by a certain value of the Gr'uneisen parameter. The specific heat is the same as in the standard Einstein model, but the pressure, equation of state, and bulk modulus can be evaluated in the MES. Using Planck oscillators, the pressure shows an anomalous behavior in terms of the volume, and the bulk modulus becomes negative for certain temperature and volume values, which is physically incorrect. When Schrödinger oscillators are used, the bulk modulus is always positive. Therefore, the different behavior of both types of oscillators indicates that only Schrödinger oscillators lead to correct results.
\end{abstract}

Keywords: Quantum harmonic oscillators; Einstein solid.

DOI: https://doi.org/10.31349/RevMexFisE.17.133

\section{Introduction}

The harmonic oscillator is ubiquitous in physics: it pops up in all the branches of the discipline, and is consequently a recurring topic in the teaching of both classical and quantum mechanics. It is in relation to the quantum harmonic oscillator that the concept of "zero-point energy" appears [1]. The zero-point energy is usually overlooked and often omitted in the calculations on the argument that it merely changes the starting point from which energy is measured and that it has no physical consequences. This is often the case, but occasionally it can be relevant; in quantum electrodynamics, for example, it is responsible for the Casimir effect [2]. In this article, we will focus on statistical mechanics, and we will show that considering or not the zero-point energy can have consequences in some cases. After Pathria [3], we will call Schrödinger oscillators those which include the zero-point energy, and Planck oscillators those which omit it. We will analyze an improved version of the Einstein solid, in which considering or not the zero-point energy is clearly important. We will show that the zero-point energy should be included to get a well-behaved bulk modulus.

The structure of this paper is the following. In Sec. 2 some results of the usual Einstein model are remembered (after all, this is an educational journal!). In Sec. 3 a modified Einstein solid (MES) is introduced and its properties are studied. Finally, in Sec. 4 the main results are gather together and discussed.

\section{The standard Einstein solid}

Einstein introduced the simplest model for solids that included characteristics of quantum mechanics [3-5]. It is as- sumed that $N$ atoms are fixed in a crystal lattice, and each can oscillate independently in the three spatial directions with a characteristic frequency $\omega$. Thus, we consider here a set of $3 N$ oscillators that share the same frequency. To perform the calculations we can use Schrödinger or Planck oscillators but the results for the specific heat are the same. For further use, we sketch the computation with both kinds of oscillators.

The energy of a Schrödinger oscillator with frequency $\omega$ is $E_{n}=(1 / 2) \hbar \omega+n \hbar \omega$, where $n$ is a positive integer that indicates the quantum state of the system; as usual $\hbar=h / 2 \pi$, with $h$ being the Planck constant. The first term is the socalled zero-point energy which is the topic of this article. In the case of a Planck oscillator, the zero-point energy is omitted, so that $E_{n}=n \hbar \omega$. If we call $k_{B}$ the Boltzmann constant as usual, the partition functions for Schödringer (subscript $S$ ) and Planck oscillators (subscript $P$ ) are:

$$
\begin{aligned}
Z_{s} & =\sum_{n=0}^{\infty} e^{-\left(E_{n} / k_{B} T\right)}=e^{-\hbar \omega / 2 k_{B} T} \\
& \times \sum_{n=0}^{\infty}\left(e^{-\hbar \omega / k_{B} T}\right)^{n}=\frac{e^{-\hbar \omega / 2 k_{B} T}}{1-e^{-\hbar \omega / k_{B} T}}, \\
Z_{p} & =\sum_{n=0}^{\infty} e^{-E_{n} / k_{B} T}=\sum_{n=0}^{\infty}\left(e^{-\hbar \omega / k_{B} T}\right)^{n} \\
& =\frac{1}{1-e^{-\hbar \omega / k_{B} T}} .
\end{aligned}
$$

From these expressions, if we have a system formed by $3 N$ oscillators, the partition function of the solid will be $Z_{P}=\left(z_{p}\right)^{3 N}$ for Planck oscillators, and $Z_{S}=\left(z_{S}\right)^{3 N}$ for Schrödinger oscillators. The internal energy $U$ in terms of the partition function $Z$ is easily obtained $[3,4]$. Consequently, 
we get two different formula for the energy depending on the kind of oscillators used:

$$
\begin{aligned}
U & =k_{B} T^{2}\left(\frac{\partial \ln Z}{\partial T}\right)_{V}, \\
U_{s} & =3 N \hbar \omega\left(\frac{1}{e^{\hbar \omega / k_{B} T}-1}+\frac{1}{2}\right), \\
U_{p} & =3 N \hbar \omega\left(\frac{1}{e^{\hbar \omega / k_{B} T}-1}\right) .
\end{aligned}
$$

Up to now, the only difference between both kinds of oscillators is a constant in the internal energy that is unobservable. From an experimental point of view the internal energy cannot be determined but the specific heat can be measured, and from (2b) and (2c) we get the same expression for the specific heat $C_{V}$ of the Einstein solid:

$$
\begin{aligned}
C_{V} & =\left(\frac{\partial U}{\partial T}\right)_{V}, \\
C_{V} & =\frac{3 N}{k_{B}}\left(\frac{\hbar \omega}{T}\right)^{2} \frac{e^{\hbar \omega / k_{B} T}}{\left(e^{\hbar \omega / k_{B} T}-1\right)^{2}} .
\end{aligned}
$$

So far there is no observable difference between both kinds of oscillators; both predict the same expression for the specific heat that agrees reasonable well with experimental data [5].

It should be noted that the partition function does not depend on volume, consequently there are many thermodynamic magnitudes of interest that are not defined. Thus, for example, the pressure or compressibility module cannot be calculated. In the next section we will improve Einstein model by introducing an explicit dependence on frequency with volume. And in this model we will see that Planck or Schrödinger oscillators yield different results.

\section{The modified Einstein solid (MES)}

A simple way to show the effect of the zero-point energy is to generalize the Einstein solid. For this, we will assume that the frequency of the oscillators depends on the volume, but first let us remember some definitions.

The Grüneisen parameter $\gamma$ can be defined in various ways $[5,6]$, but the definition that better suits our purposes is the following:

$$
\gamma=-\frac{V}{\omega} \frac{\partial \omega}{\partial V}
$$

Much can be said about the Grüneisen parameter, but for our aim it is a measure of the change in the oscillatory frequency $\omega$ when the volume $V$ of the solid changes ${ }^{i}$. The relations of this parameter with thermodynamic and microscopic properties of a solid have been explored elsewhere [7,8].

Here we can introduce a model, which we will call the modified Einstein solid (MES), where the frequency is a function of the volume $\omega(V)$ and which is characterized by a certain value of the parameter $\gamma$. The simplest assumption is to take the Grüneisen parameter as a constant. If $\gamma$ is constant, then Eq. (4) can be easily solved and we get $\omega=C V^{-\gamma}$ where $C$ is an integration constant that can be evaluated as follow. If $\omega_{0}$ is the frequency of the solid when it is subjected to atmospheric pressure $p_{0}$ and its volume is $V_{0}$, we can get an expression for $C$ and finally obtain:

$$
\omega=\omega_{0}\left(\frac{V}{V_{0}}\right)^{-\gamma} .
$$

Notice that this dependence on frequency with volume implies that the frequency decreases as the volume increases. This is a behavior found in other well-known oscillatory system. Remember that the frequency of a simple pendulum goes as $L^{-1 / 2}$ where $L$ is the length of cord i.e. the frequency diminishes with an increase of the system size.

It should be remarked that Eq. (5) does not modify the result of the specific heat. However, the pressure can now be evaluated with the usual formula:

$$
P=k_{B} T\left(\frac{\partial \ln Z}{\partial V}\right)_{T}
$$

At this point, it is useful to introduce dimensionless variables that will facilitate subsequent analysis. A characteristic temperature $\theta_{0}$ is defined as $\theta_{0}=\hbar \omega_{0} / k_{B}$ and based on it a dimensionless temperature $t$ can be introduced ${ }^{i i}$. Likewise, if $V_{0}$ is the volume of the solid at atmospheric pressure, a dimensionless volume $v$ can be defined. In mathematical terms:

$$
\begin{aligned}
\theta_{0} & =\frac{\hbar \omega_{0}}{k_{B}}, \\
T & =t \theta_{0}, \\
V & =v V_{0} .
\end{aligned}
$$

From Eq. (6) two pressures are obtained, a $P_{S}$ if Schödringer oscillators are used and a $P_{P}$ if Planck oscillators are utilized. Two state equations are thus obtained for this solid according to the type of oscillator used:

$$
\begin{aligned}
P_{s} & =\frac{\left(1+e^{v^{-\gamma} / t}\right) v^{-1-\gamma} \gamma}{2\left(e^{v^{-\gamma} / t}-1\right)} \frac{3 N k_{B} \theta_{0}}{V_{0}}, \\
P_{P} & =\frac{v^{-1-\gamma} \gamma}{\left(e^{v^{-\gamma} / t}-1\right)} \frac{3 N k_{B} \theta_{0}}{V_{0}}
\end{aligned}
$$

It is interesting to write the equation of state of this solid in a compact form. Thus after some algebraic manipulations, it comes out that:

$$
\begin{aligned}
& P_{s}=\frac{U_{s} \gamma}{V}, \\
& P_{p}=\frac{U_{p} \gamma}{V} .
\end{aligned}
$$




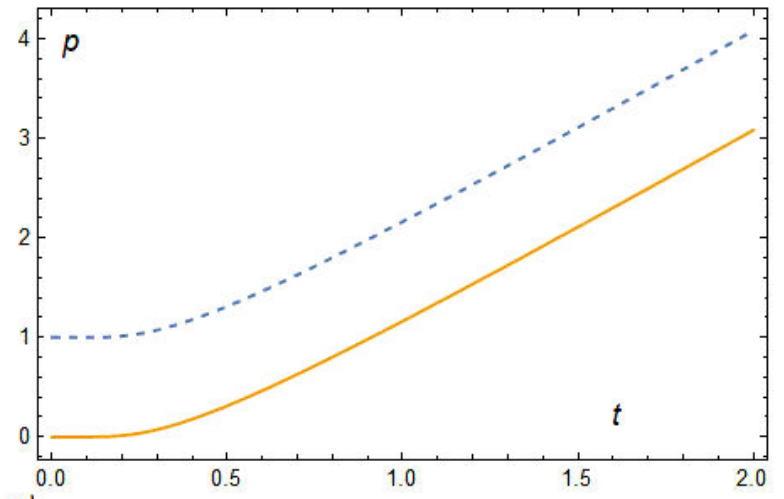

a)

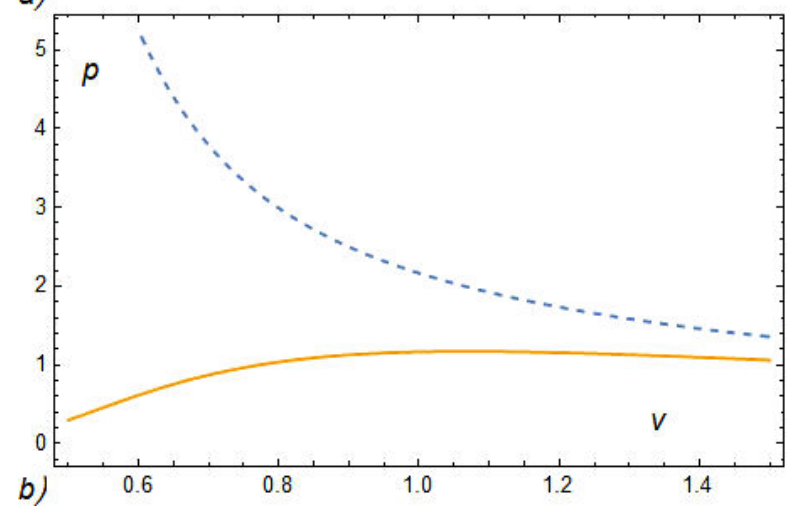

FIGURE 1. The dimensionless pressure $p$ is shown here in terms of dimensionless temperature $t$ (a) and dimensionless volume $v$ (b). It has been taken $\gamma=2$ which is a typical value of the Grüneisen parameter [9]. The solid line corresponds to the Planck oscillators while the dashed line corresponds to the Schrödinger oscillators. In (a) it has been taken $v=1$ while in (b) it has been taken $t=1$. Observe the anomalous behavior of the pressure below $v \approx 1$ when using Planck oscillators: the pressure decreases as the volume decreases. This is not right. On the other hand, with Schrödinger oscillators the behavior is normal: higher pressures are necessary to compress the solid.

$U_{S}$ and $U_{P}$ are given by Eqs. (2b) and (2c) but taking into account that now the frequency $\omega$ depends on the volume as shown in Eq. (5). Once again, both kinds of oscillators lead to the same result.

It is appropriate to define a dimensionless pressure $p$ as: $P=p 3 N k_{B} \theta_{0} / V_{0}$. Figure 1 shows the dimensionless Schrödinger (dashed line) and Planck (solid line) pressures in terms of the dimensionless temperature $t$ (Fig. 1a) and volume $v$ (Fig. 1b). It has been taken $\gamma=2$ which is a typical value for the Grüneisen parameter as can be seen from experimental data [9].

Note the anomalous behavior of the pressure in the case of Planck oscillators: as the volume decreases below $v \approx 1$, the pressure decreases ${ }^{i i i}$. This result is contrary to our physical intuition: the pressure is expected to increase to decrease the volume. And this is exactly the behavior when using Schrödinger oscillators. With this result we could conclude our task: it has been shown that using Planck oscillators in this modified Einstein solid, a non-physical result is obtained, while using Schrödinger oscillators everything works correctly. But it will also be shown that both types of oscillators lead to different mechanical properties.
The bulk modulus $K$ is given by:

$$
K=-V\left(\frac{\partial P}{\partial V}\right)_{T}
$$

And if we remember that the pressure can be evaluated in terms of the partition function $Z$ as shown in (6), it turns out that:

$$
K=-K_{B} T V\left(\frac{\partial^{2}(\ln Z)}{\partial V^{2}}\right)_{T} .
$$

At this point, we find that there are two different bulk moduli: $K_{P}$ for Planck oscillators and $K_{S}$ for Schrödinger oscillators:

$$
\begin{aligned}
& K_{s}=-3 N K_{B} T V\left(\frac{\partial^{2}\left(\ln Z_{s}\right)}{\partial V^{2}}\right)_{T}, \\
& K_{s}=-3 N K_{B} T V\left(\frac{\partial^{2}\left(\ln Z_{p}\right)}{\partial V^{2}}\right)_{T} .
\end{aligned}
$$

All is left to do now is to replace the expressions $z_{S}$ and $z_{P}$ given by Eq. (1a) and (1b) in Eq. (12) and to perform the calculation. This gives:

$$
\begin{aligned}
K_{s} & =\frac{3 N k_{B} \theta_{0}}{V_{0}}\left(\frac{e^{2 v^{-\gamma} / t} v^{-1-\gamma}(1+\gamma) \gamma}{2\left(e^{\left(v^{-\gamma} / t\right)}-1\right)^{2}}\right. \\
& \left.-\frac{v^{-1-\gamma}(1+\gamma) \gamma}{2\left(e^{v^{-\gamma} / t}-1\right)^{2}}-\frac{e^{\left(v^{-\gamma} / t\right)} v^{-1-2 \gamma} \gamma^{2}}{\left(e^{v^{-\gamma} / t}-1\right)^{2} t}\right) \\
K_{p} & =\frac{3 N k_{B} \theta_{0}}{V_{0}}\left(\frac{e^{\left(v^{-\gamma} / t\right)} v^{-1-\gamma}(1+\gamma) \gamma}{\left(e^{v^{-\gamma} / t}-1\right)^{2}}\right. \\
& \left.-\frac{v^{-1-\gamma}(1+\gamma) \gamma}{\left(e^{v^{-\gamma} / t}-1\right)^{2}}-\frac{e^{2 v^{-\gamma} / t} v^{-1-2 \gamma} \gamma^{2}}{\left(e^{v^{-\gamma} / t}-1\right)^{2} t}\right) .
\end{aligned}
$$

The two expressions are clearly different; there are factors " 2 " in the $K_{s}$ formula that are absent in $K_{p}$. This means that, when a volume-dependent frequency is introduced, the use of Planck or Schrödinger oscillators leads to different results which can be observed quite easily because measuring the bulk modulus is a routine experiment (see [10] and references therein). Anyway, the MES is too simple of a model to allow a detailed comparison with experimental data.

It is convenient to define a dimensionless bulk modulus $k_{S}$ and $k_{P}$ as: $K_{S}=k S 3 N k_{B} \theta_{0} / V_{0}$ and $K_{P}=$ $k P 3 N k_{B} \theta_{0} / V_{0}$. Figure 2 shows $k_{S}$ (dashed line) and $k_{P}$ (solid line) as a function of the dimensionless temperature $t$ (Fig. 2a) and of the dimensionless volume $v$ (Fig. 2b) using a Grüneisen parameter $\gamma=2$.

The significant outcome from Fig. 2 is that the bulk modulus becomes negative when Planck oscillators are used, and this is physically impossible. From this it can be inferred that the use of Planck oscillators leads to incorrect results. Instead, with Schrödinger oscillators, the bulk modulus results positive in the entire range of temperatures and volumes, as it should be. 

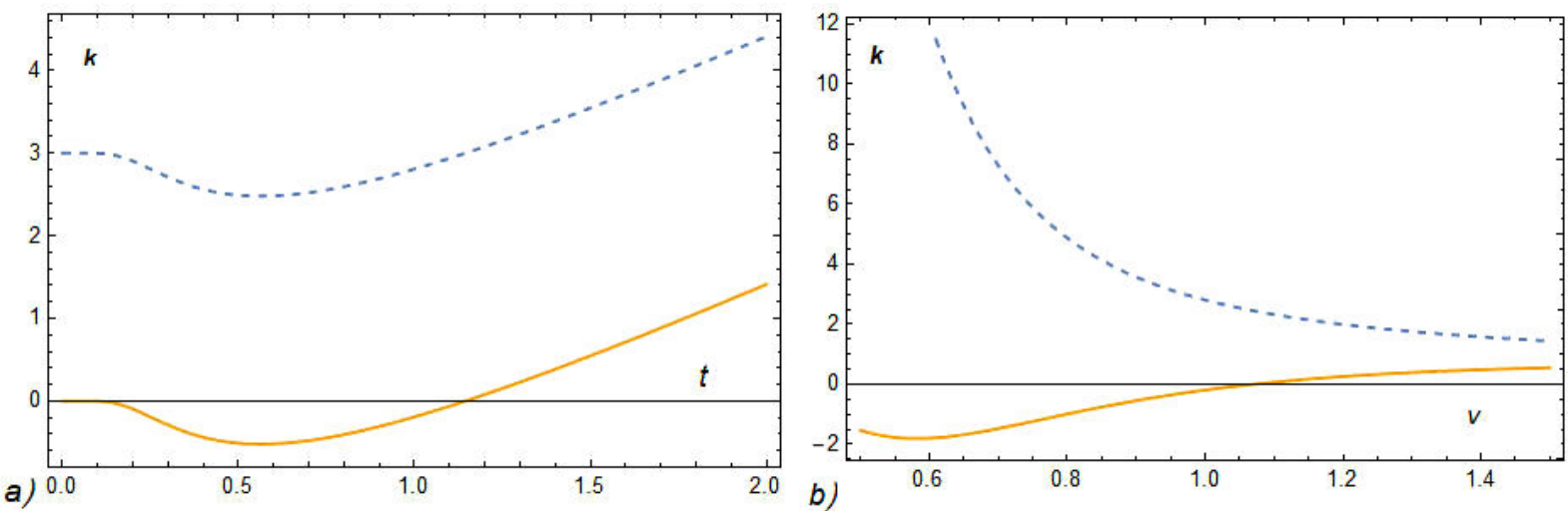

FIGURE 2. a) The dimensionless bulk modulus $k$ as a function of the dimensionless temperature $t$ and b) of the dimensionless volume $v$. The solid line is obtained from Planck oscillators, while the dashed line corresponds to Schrödinger oscillators. As it is a typical value, a Grüneisen parameter $\gamma=2$ was used. In (a) the case for a fixed volume $v=1$ is considered, and in (b) for a fixed temperature $t=1$. Note in both cases that $k$ becomes negative for Planck oscillators, and that is physically incorrect. The result that makes physical sense is obtained with Schrödinger oscillators.

\section{Conclusions}

The intention in this article has been to show the physical implications of taking into account or not the zero-point energy of an oscillator. The term $1 / 2 \hbar \omega$ in the energy of a quantum oscillator is not irrelevant.

We have considered a modified Einstein solid (MES), characterized by a certain characteristic temperature $\theta_{0}$ and a certain value for the Grüneisen parameter $\gamma$, i.e. this is a solid where the frequency of the oscillators changes with the volume. Our analysis has shown that:

a) The specific heat of the MES is the same as that of the usual Einstein solid.

b) The MES equation of state is given by Eq. (9) and it is formally the same for both kinds of oscillators.

c) The relation between pressure and volume shows an unusual behavior if Planck oscillators are used.

d) The bulk modulus becomes negative for certain ranges of volume and temperature if Planck oscillators are used. This result is unphysical. If Schrödinger oscillators are considered, the bulk modulus is always positive.

In summary, a simple generalization of the Einstein model has been introduced and it has been shown that it is not the same to use Planck or Schrödinger oscillators.

Finally, it should be noted that some statistical mechanics textbooks omit the zero-point energy when dealing with the harmonic oscillator and the Einstein solid, both in the canonical and the microcanonical ensembles. And surely, many university lecturers (the present author included) also omit it. But in this article, such a practice has been proven incorrect. Although there are no differences in the common results, when we look into subtler questions, differences do arise. Certainly, Schrödinger and Planck oscillators do not lead to the exact same physics.

\section{Acknowledgments}

The author thanks the unknown referee for careful reading the manuscript, checking the calculations and making suggestions that improved the final version of the article. $i$. In a more advanced treatment, the Einstein model [3,4,5] is seen as a set of phonons, all of them with the same frequency. An electromagnetic analogy can help to understand the meaning of the Grüneisen parameter. Consider a resonant microwave cavity like that is used in microwave ovens. The resonant frequency of the cavity depends on its physical dimensions. A change in the size of the resonant cavity means a change in the resonant frequency and therefore in the frequency of the photons there. The Grüneisen parameter describes this frequency change with size for mechanical waves and its associated particles (phonons). ii. Notice $\theta_{0}$ that would be the Einstein temperature if the frequency $\omega$ was be constant.

iii. The pressure $p_{P}$ reaches its maximum value at $v=1.0695$ as it was kindly noticed by the article referee. Below that value, the behavior of the pressure with the volume is anomalous.

1. D. J. Griffiths and D. F. Schroeter, Introduction to Quantum Mechanics, 3rd ed. (Cambridge University Press, Cambridge, 2018), https://doi.org/10.1017/ 9781316995433 
2. Wikipedia, "Casimir Effect", Wikipedia.org, https://en. wikipedia.org/wiki/Casimir_effect

3. R. K. Pathria, Statistical Mechanics, 2nd ed. (ButterworthHeinemann, Oxford, 1996), https://doi.org/10. 1016/B978-0-7506-2469-5.X5000-2

4. R. H. Swendsen, An Introduction to Statistical Mechanics and Thermodynamics, (Oxford University Press, Oxford, 2012).

5. C. Kittel, Introduction to Solid State Physics, 8th ed. (Wiley, New York, 2004).

6. Wikipedia, "Grüneisen parameter", Wikipedia.org, https://en.wikipedia.org/wiki/Gr|unhbox〉 voidb@x\bgroup let lunhbox \voidbex\setbox etempboxa \hbox $\{u \backslash g l o b a l \backslash$ mathchardef $\backslash$ accentespacefactor\spacefactor\} \accent 127u〉egroup\spacefactor\accente spacefactorneisen_parameter
7. D. S. Bertoldi, E. N. Miranda, and A. F. Guillermet, Revisiting the thermostatistics of the Grüneisen parameters and applications to quasiharmonic solids, J. Phys. Chem. Solids 75 (2014) 1147, https : //doi.org/10.1016/j.jpcs . 2014.03 .015

8. F. D. Stacey and J. H. Hodgkinson, Thermodynamics with the Grüneisen parameter: Fundamentals and applications to high pressure physics and geophysics, Phys. Earth Planet. Inter. 286 (2019) 42, https://doi.org/10.1016/j.pepi. 2018.10 .006

9. R. A. MacDonald and W. M. MacDonald, Thermodynamic properties of fcc metals at high temperatures, Phys. Rev. B $\mathbf{2 4}$ (1981) 1715, https : //doi.org/10.1103/PhysRevB. 24.1715

10. M. de Jong et al., Charting the complete elastic properties of inorganic crystalline compounds, Sci. Data 2 (2015) 150009, https://doi.org/10.1038/sdata.2015.9 